\title{
NIÑEZ Y JUVENTUD EN SITUACIÓN DE RIESGO: LA GESTIÓN SOCIAL DEL RIESGO. Una revisión bibliográfica
}

\author{
PATRICK DONOVAN ${ }^{*}$ \\ XIMENA OÑATE* \\ GONZALO BRAVO ${ }^{* * *}$ \\ M. TERESA RIVERA ***
}

\begin{abstract}
RESUMEN
Este artículo observa como las grandes transformaciones (económicas, políticas y culturales) contemporáneas impactan en la vida cotidiana de la familia, niñez y juventud. Desde aquella problemática, se explora la literatura de las ciencias sociales en torno al concepto de gestión del riesgo social, más específicamente en el ámbito de la niñez y juventud en situación de dificultad. De hecho, la teoría del riesgo se ha convertido hoy en día en una gran preocupación, quizás exagerada y políticamente interesada.

Esta observación documental recoge y contrasta información de materiales predominantemente del Reino Unido, América Latina y Chile. Parte de una revisión histórica del concepto de riesgo, identifica una serie de esquemas teóricos para abordarlo, y luego se centra en el debate de la gestión del riesgo con relación a la niñez en situación de dificultad.

Se observa que hace falta aún un modelo de intervención social del riesgo. Sin embargo, se identifica un continuo entre dos grandes tendencias: una que se centra en el control y busca evitar el riesgo, mientras que la otra enfatiza la participación y el empoderamiento, tomando riesgos positivos. Se incluyen interrogantes para investigaciones futuras en este ámbito.

\section{PALABRAS CLAVE: INTERVENCIÓN SOCIAL, RIESGO, MODELO DE GESTIÓN SOCIAL DE RIESGO}

\footnotetext{
* Profesor e investigador, Departamento de Ciencias Sociales, Universidad de La Frontera, Temuco, Chile.

** Profesora e investigadora, Departamento de Psicología, Universidad de La Frontera.

*** $\quad$ Profesor e investigador, Universidad de La Frontera.

**** Coordinadora de la Red de Apoyo Espiritual Cristiano a la Infancia en Dificultad (RAECID).
} 


\title{
RESUMO
}

Este artigo observa como as grandes transformações (econômicas, políticas e culturais) contemporâneas influenciam a vida cotidiana da família, da infância e da juventude. A partir daquela problemática, explora-se a literatura das ciências sociais sobre o conceito de gestão do risco social, mais especificamente no âmbito da infância e juventude em situação difícil. De fato, a teoria do risco transformou-se atualmente numa grande preocupação, talvez exagerada e de interesses políticos. Esta observação documental reúne e contrasta informações de materiais em sua maioria do Reino Unido, da América Latina e do Chile. Parte de uma revisão histórica do conceito de risco, identifica uma série de esquemas teóricos para abordá-lo, e em seguida se concentra no debate da gestão do risco com relação à infância em situação difícil. Observa-se que faz falta ainda um modelo de intervenção social do risco. Contudo, identifica-se um contínuo entre duas grandes tendências: uma com enfoque no controle e que busca evitar o risco, enquanto a outra enfatiza a participação e o empoderamento, tomando riscos positivos. Inclui-se desafios para investigações futuras neste âmbito.

\section{PALAVRAS CHAVE: INTERVENÇÃO SOCIAL, RISCO, MODELO DE GESTÃO SOCIAL DE RISCO}

\begin{abstract}
This article observes the way in which great contemporary transformations (economic, political and cultural) have an impact in the daily life of family, childhood and youth. Toward the problematic area, the literature of social sciences around the concept of management of social risk is explored, more specifically in the scope of childhood and youth in a difficult situation. In fact, the theory of the risk has nowadays become a great preoccupation, perhaps exaggerated and politically interested. This documentary observation predominantly collects and resists of materials of the United Kingdom, Latin America and Chile. Part of an historical revision of the risk concept identifies a series of theoretical schemes to approach it, and soon it is centered in the debate of management of the risk in relation to childhood in a difficult situation. It is observed that there is still lack of a model for social intervention of risk. Nevertheless, a continuation between two great tendencies is identified: one that is centered in control and looks to avoid the risk, whereas the other emphasizes the participation and the empowerment, taking positive risks. Questions for future investigations in this scope are included.
\end{abstract}

KEY WORDS: SOCIAL INTERVENTION, RISK, A MODEL FOR SOCIAL INTERVENTION 


\section{INTRODUCCIÓN}

EN EL TRANSCURSO DE los dos últimos decenios, las economías y sociedades de los países desarrollados y en desarrollo han vivido procesos de cambio estructural de amplitud y profundidad considerables (Alburquerque, 1999:9). Se advierten «grandes cambios, más que cambios, una mutación del tipo de sociedad que pasa de una sociedad industrial a una postindustrial, de una sociedad de la energía a una de la información» (Touraine, 2001:30), lo que se califica a la vez como una época de oportunidades y riesgos peculiares.

A pesar de las oportunidades que brinda la nueva sociedad en la cual vivimos, varios estudios enfatizan sus amenazas dado que, «en la modernidad avanzada, la producción social de la riqueza se relaciona en forma sistemática con la producción social del riesgo» (Beck, 1992:19; Giddens, 2000:97), en particular con el advenimiento de la globalización que, «en su encarnación de capitalismo informacional, desregulado y competitivo, supera a los estados, pero articula a los segmentos dinámicos de las sociedades de todo el planeta, al tiempo que desconecta y margina a aquellos que no tienen otro valor que el de su vida» (Castells, 1999:3).

En este contexto de grandes transformaciones (económicas, políticas y culturales), se constata que «los cambios registrados en los últimos años afectan especialmente la vida cotidiana de la gente y su sociabilidad, con sus tejidos familiares y comunitarios, sus valores e identidades» (PNUD, 1998:15). La familia, vista tradicionalmente como una fuente de seguridad, es también amenazada. La familia es cada vez menos eficaz para gestionar sin desintegrarse los desafíos que se le presentan, tales como la incorporación de la mujer al trabajo, la creciente individuación de las preferencias y estilos comunicativos de los hijos, las exigencias económicas para la integración por medio consumo, convirtiéndose en situaciones generalizadas de inseguridad (PNUD, 1998:26).

Estos cambios estructurales en el desarrollo actual del capitalismo y de nuestra sociedad marcadamente neoliberal produce entonces un contexto específico que afecta el desarrollo de la familia, de la niñez y de la juventud (Balardini, 2000:20-22), en particular en cuanto a la niñez y juventud que vive en condiciones deficientes de desarrollo (Unicef, 2002).

De hecho, no existe una sola niñez y juventud en Chile como nos advierte el historiador Gabriel Salazar (2002:11), sino varias, según la 
heterogeneidad socioeconómica y la desigualdad cultural de nuestra sociedad. Salazar, con relación al siglo XIX, distingue una «jeunesse dorée ('caballeritos')» y una «jeunesse de la galère ('cabros de la ca1le', 'huachos', 'pelusas', etc.)». Con relación a la época presente, Touraine (1990:2) se refiere a dos imágenes de juventud: la juventud rosada, o sea la juventud instrumento de la modernización, y la juventud negra, o sea la juventud percibida como elemento marginal y hasta peligroso. Se refiere a esta última categoría como a los jóvenes sin empleo, que vienen con frecuencia de familias «rotas», los jóvenes que logran sobrevivir gracias a trabajos intermitentes y mal pagados, a actividades no declaradas, y que son propensos a la delincuencia.

Se enfoca entonces a la niñez y a la juventud como sujetos sociales y culturales heterogéneos productos de nuestra sociedad actual, que viven situaciones sociales diversas e identidades múltiples, y que van construyendo su vida según cuotas diversas de riesgo social.

Si abordamos la situación de riesgo en Chile desde la perspectiva de la Seguridad Humana, se plantea que, aún si nuestro país ha tenido desde los años noventa importantes logros económicos y sociales, ${ }^{1}$ sin embargo «la Seguridad Humana, tanto objetiva como subjetiva, es baja y está desigualmente distribuida entre los distintos grupos sociales y regiones del país. Ambos índices calculados dan fundamento empírico a esta afirmación» (PNUD, 1998:27). El Informe describe como se vive esta inseguridad en ámbitos particulares tales como la salud y la previsión, la educación, el trabajo y el consumo, desde la cotidianidad y trayectorias de familias, destacando cómo son afectados los jóvenes en esta situación, no solamente incorporando pautas y valores como drogadicción y alcoholismo, sino también llegando a la ruptura con el proyecto familiar y la construcción de lazos intergeneracionales.

En cuanto a la delincuencia infanto-juvenil a nivel nacional, desde 1994 al 2005, se observa que, de las 1.993 .977 personas que fueron aprehendidas, un 36\% son niños entre 10 y 18 años, siendo el robohurto el delito más frecuente. Se observa también que la evolución del índice de adultos e infanto-juveniles aprehendidos en Chile del $2000 \mathrm{al}$ 2005 según delitos (daños, drogas, homicidio, hurtos, lesiones, robos y violación) se incrementa, pasando en el caso de los adultos de 99.245 a

1 Entre otros, cabe señalar: una alta tasa de crecimiento, una fuerte disminución de la pobreza y una activa y concreta preocupación por los grupos vulnerables desde distintos puntos de vista: el espacial, el etario, el socioeconómico y el de género (PNUD, 1998:16). 
152.251 (un índice de incremento de $153 \%$ ), y, en el caso de los niños de 5 a 19 años, de 82.029 a 91.850 (un índice de incremento de 112\%) (Gaete y Lara, 2007).

En Chile, el Servicio Nacional de Menores (Sename) es el organismo de Estado que tiene por misión contribuir a proteger y promover los derechos de los niños, niñas y adolescentes que han sido vulnerados en el ejercicio de los mismos y a la inserción social de adolescentes que han infringido la ley penal. Esta labor se desarrolla a través de la oferta de programas especializados en el área de Protección de Derechos y en el área de Responsabilidad Juvenil, en coordinación con actores públicos (Ministerio de Justicia y otros servicios públicos del Estado) y privados (Sename, 2004; 2005a; 2005b y 2006).

El lograr la inclusión social de la niñez y de la juventud así como su pleno desarrollo plantea un gran desafío a la sociedad y al Estado chileno. Aún mayor es el desafío para lograr la rehabilitación y reinserción de quienes han sido atropellados en sus derechos por abandono familiar, violación física, violación emocional y abuso sexual o que se han convertido en niños y jóvenes delincuentes (Donovan, Oñate, Bravo y Rivera, 2006).

La precaria capacidad del actual modelo de desarrollo de distribuir oportunidades en forma equitativa y niveles de vida con seguridad humana, así como de alcanzar la reinserción social de la niñez y juventud vulnerados desafían a los académicos de las ciencias sociales y nos mueve a indagar en torno a cómo superar el riesgo social que produce nuestro sistema.

Surge entonces el debate académico y profesional en ciencias sociales en torno a cuales serían las estrategias más adecuadas para proteger y promover los derechos de la niñez y juventud, ${ }^{2}$ en especial aquellas que han sido vulneradas en sus derechos. Enmarcado en este contexto, este estudio revisa el concepto de riesgo social, vinculado sistemáticamente con nuestro sistema socioeconómico de producción, y los aportes científicos en este debate en torno a cómo enfrentar en forma óptima la gestión de dicho riesgo, en particular con relación a la niñez y juventud en el contexto actual.

La finalidad de este artículo es de explorar la literatura sobre la gestión del riesgo y la incertidumbre en la intervención social para identifi-

2 En este estudio, en cuanto a rango de edad, se considera niñez de 0 a 18 años, rango que utiliza la Convención Internacional de los Derechos del Niño; y juventud de 18 a 25 años. 
car sus principales hallazgos, desafíos y argumentos, así como discutir puntos de consenso y de disenso. El artículo se centra en la gestión del riesgo más que en la ocurrencia del riesgo, prestando mayor atención a la literatura sobre la intervención social a nivel de la infancia y juventud en situación de dificultad.

Este artículo explora literatura del Reino Unido, de América Latina y de Chile sobre la temática, pero no en forma exclusiva, con un énfasis en el trabajo teórico e investigaciones publicadas, y no tanto en documentos sobre políticas y prácticas específicas. Los documentos fueron identificados en forma privilegiada, pero no exclusiva, mediante la observación de la base de datos de las publicaciones Sage (septiembre y octubre 2007), una editorial que reúne cerca de 30 revistas en torno a las ciencias sociales.

Se aborda la temática en tres momentos. Se parte introduciendo la emergencia del concepto de riesgo, centrándose en las principales definiciones que han influenciado la intervención social. En una segunda parte se describe brevemente las dimensiones teóricas del concepto. Finalmente se estudian diversas estrategias en relación a la gestión del riesgo.

\section{LA EMERGENCIA Y DESARROLLO DEL CONCEPTO}

Riesgo es un concepto complejo y multifacético (Ryan, 1996; Stevenson, 1999; Warner, 1992) nos señala Stalker (2003:211-212), identificando literatura sobre el tema tanto en medicina, salud pública, ingeniería, estudios de ciencias comerciales, derecho y seguros económicos.

Con relación a la emergencia del concepto de riesgo con relación a la conducta humana, Stalker (2003:214) hace la recensión de varios autores que abordan el tema tales como Jaeger, Luhmann, Douglas, Parton, Lupton, Brearley, Warner, Shaw y Shaw. En cuanto a Jaeger y otros (2001), identifican la idea de gestión del riesgo en el código de Hammurabi. Luhmann (1993) encuentra una referencia al riesgo en el siglo XVI en la literatura alemana vinculada a los seguros marítimos y referidos al cálculo de la probabilidad de diversos tipos de desastres marítimos. Douglas (1992) y Parton (1996) observan literatura en el siglo XVII relacionada con el concepto de probabilidad de la ocurrencia de un evento y sus consecuencias en cuanto a ganancias o/y daños.

Por su parte Lupton (1999:7) advierte el cambio de creencias vinculadas al riesgo con la aparición de las estadísticas y el cálculo de las 
probabilidades, lo que lleva al estudio científico del riesgo, distinguiendo entre riesgo, «condición en la cual la estimación probable de un evento es conocido o conocible», e incertidumbre «cuando las probabilidades no se pueden estimar». Warner (1992:2) advierte que en el siglo XIX el riesgo se vincula más y más a eventos o desenlaces no deseados, lo que prevalece aún hoy en día.

Al concepto de riesgo, Jaeger (2000:17) incorpora al ser humano: o como víctima o como agente. Brearley (1982:82) distingue entre el peligro, relacionado al miedo de la ocurrencia de un evento que puede causar un daño, y la vulnerabilidad, «la relativa variación en posibles desenlaces negativos». Incorpora también la idea de «factores que pueden reducir desenlaces peligrosos» (Stalker, 2003:213).

El concepto de vulnerabilidad planteado por Brearley $(1982: 26,82)$ tiene su importancia. Si bien el riesgo puede alcanzar a todos, sin embargo existen grupos humanos más vulnerables al riesgo, con menos capacidades para reducir el desenlace negativo del peligro (Brearley, 1982:26,82) y más susceptible de ser víctimas de un daño significativo (Sargent, 1999) como la niñez, la adolescencia y la juventud, aún si dichas categorías sociales se pueden convertir en amenaza para la sociedad cuando atropellan los derechos de otros.

Múltiples autores (Parton, 1996; Lupton, 1999; Shaw y Shaw, 2001; citado por Stalker, 2003:214) observan que el concepto de riesgo se ha convertido en un término de uso amplio en la sociedad occidental contemporánea donde se tiende a identificar riesgo con incertidumbre (Lupton, 1999:9). Se advierte también que riesgo no es un concepto legal preciso, lo que deja gran margen de interpretación en los procedimientos judiciales, conllevando gran incertidumbre en cuanto a poder establecer normas precisas de responsabilidad social, lo que complica la toma de decisiones en trabajo social (Stalker, 2003:214).

\section{DIMENSIONES TEÓRICAS}

Para abordar el estudio teórico del concepto de riesgo, Jaeger y otros (2001) mencionan diversas disciplinas que abordan el tema: las estadísticas, la psicología, la sociología, la antropología, la geografía. Se pueden añadir otras disciplinas tales como la psicoeducación y las ciencias políticas.

En el campo de las estadísticas, el riesgo se enfoca desde la perspectiva del cálculo de las probabilidades, es percibido como un hecho 
objetivo y es considerado como la base de la aproximación científica contemporánea (Stalker, 2003:214).

La psicología aborda también el tema, analiza estos riesgos (negativos) o daños significativos (Sargent, 1999; Stalker, 2003:214) que enfrenta el individuo en la sociedad moderna. En cuanto a la niñez y la juventud en riesgo se elabora la siguiente tipología: riesgos o daños en relaciones abusivas (abuso sexual, maltrato infantil, negligencia), comportamientos autodestructivos (alcoholismo, drogadicción, prostitución, vagancia y mendicidad) y desadaptación social (desadaptación escolar, comportamientos violentos, trastornos de conducta, pandillaje, conflictos con la justicia, trastornos sexuales como pedofilia) (PerezLuco, S/F:18).

Estos problemas de relaciones abusivas, de comportamientos autodestructivos y desadaptados se suelen enfocar en psicología social y en sociología como el resultado de una interacción compleja entre rasgos de personalidad con lo social (v. g. la familia, el grupo de pares), con el entorno específico (v. g. escuela, hogar) y según los factores de riesgo de la vecindad (Sprott, Jenkin y Doob 2005:59; Farrington, 1998; Gottfredson y Hierschi, 1990; Klein, Forehand, Armistead y Long, 1997; Loeber y Farrington, 1998; Samson y Lamb, 1993).

La psicología ha sido muy influenciada por el enfoque de la psicometría que estudia la ocurrencia del riesgo a partir de la teoría de la conducta racional (Lupton, 1999; Stalker, 2003:214). Cabe señalar que se observan críticas al enfoque de la psicometría por no considerar la dimensión simbólica del riesgo y sus significados sociales, por aislar al individuo de su contexto social y separar un riesgo particular o un comportamiento de riesgo del contexto de riesgos y comportamientos asociados (Stalker, 2003:214).

La psicoeducación aborda también el estudio del riesgo social. Es una disciplina profesional que desarrolla a través de los años un modelo relacionado con la atención a jóvenes en riesgo (Desrochers y Dionne, 2008:149). La psicoeducación «se basa en una filosofía humanista, la que se manifiesta en la aplicación y elaboración de un modelo terapéutico, la organización de un ambiente físico, la creación de instrumentos pedagógicos apropiados, la búsqueda de un apoyo científico para la acción educativa y una formación específica de los educadores» (Renou, 1989; citado por Desrochers y Dionne, 2008:149).

El enfoque teórico de la desviación social es una aproximación teórica que se anticipa a la teoría del riesgo y que estudia el fracaso de 
la sociedad en regular el comportamiento humano. En Chile, la socióloga Doris Cooper estudia la desviación y delincuencia juvenil, la delincuencia urbana femenina actual, la teoría del continuum subcultural de la delincuencia, las características culturales de la criminalidad mapuche.

En el campo de los estudios recientes en sociología y antropología sobre riesgo social se cuestiona la idea de la modernidad como un tiempo de progreso continuo. La modernidad es más bien enfocada como un tiempo de cambio, rupturas e incertidumbre ${ }^{3}$ (Giddens, 1990; Beck, 1992, citado por Stalker, 2003:214) que inclusive incorpora una dimensión global, lo que revela la complejidad de los cambios en los procesos sociales actuales en curso y la necesidad de acudir a la asesoría de expertos, aún si éstos no siempre coinciden entre si, ni son infalibles.

Douglas (1982; 1986; 1992, citado por Stalker, 2003:215) define el riesgo como una estrategia para enfrentar los peligros que se perciben. Recalca las variaciones en cuanto a la percepción y valoración de los peligros. Lo atribuye a las diferencias socioculturales y políticas de los actores sociales, tanto en cuanto a los actores expertos como a otros. Cabe añadir que esta autora es crítica con relación al concepto de riesgo, destacando que a menudo el carácter científico del concepto es precario, argumentando que en casi toda situación de riesgo se vinculan sentimientos de ira, esperanza y temor (1992:12).

Noceti (2005:17-18), antropóloga argentina, prolonga la crítica planteada por Douglas y enfatiza la ambigüedad del concepto de riesgo ya que éste puede ser interpretado en forma arbitraria e ideológica. Noceti devela como dicho concepto tiene una historia específica, que incluye el ámbito de la política pública, pero que atraviesa también diversas esferas sociales en donde se construyen discursos y prácticas, a la vez que se moldean dispositivos (Foucault, 1995) acordes a objetivos socialmente planteados (Noceti, 2005:17). «Riesgo es un sustan-

3 Esta percepción varía según los autores: Giddens no percibe este tiempo como de mayores riesgos que antes, mientras que Beck no duda que se vive en una sociedad de mayores riesgos que antes y define la sociedad como «la sociedad (industrial) del riesgo». Jaeger y otros (2001:9) perciben que el riesgo es «una nueva criatura que aparece al final del siglo veinte». Acorde a Beck (1992), vivimos en una «sociedad del riesgo», donde las posibilidades de un riesgo positivo, «bueno» (tomando riesgo para alcanzar un desenlace positivo) es superado por el riesgo percibido casi en forma exclusiva como una amenaza, un azar, un daño o un peligro (Case, 2007:92; Lupton, 1999:8). 
tivo, pero 'en riesgo' es una frase que funciona como adjetivo calificativo de ciertos sujetos que, dadas sus condiciones de vida, ponen en peligro el orden social establecido, edificado sobre ideas determinadas de moral y justicia, se involucra en un sistema ético determinado que cataloga ciertos aspectos sociales como peligrosos al mismo tiempo que ignora otros» (Douglas, 1996, citado por Noceti, 2005:18).

Noceti observa entonces que el concepto de riesgo puede ser utilizado en forma ideológico-política, percibiendo a ciertos sujetos sociales meramente como amenaza a la sociedad, sin considerar que la misma sociedad, por el sistema económico que prevalece y la falta de protección social del Estado hacia aquellos sectores sociales vulnerables, participa en la causalidad de su desviación social. Reivindica que, allí donde la niñez padeciera una situación de supervivencia, «se hace imperiosa la actuación del Estado como garante de la Protección Integral de sus niños» (Noceti, 2005:18).

Jaeger y otros (2001; Stalker, 2003:216) abordan el concepto de riesgo desde un marco más amplio de la teoría social. Argumentan que el Paradigma del Actor Racional (PAR en español y RAP en inglés), ha dominado el mundo moderno occidental. Sin embargo el actual riesgo pone en tela de juicio aspectos de la seguridad ontológica que plantea Giddens: los cambios en las identidades sociales, la fragilidad de la organización y convivencia de la sociedad contemporánea, y el riesgo de destrucción del planeta. Plantea que esta situación no puede ser explicada a cabalidad por el paradigma vigente ya que éste no toma suficientemente en consideración la macrosociología y las fuerzas históricas por una parte, y no contempla las dimensiones individuales no-racionales por otra parte. Los autores sugieren abordar el tema explorando la potencialidad de otras teorías sociales tales como la modernización reflexiva, la teoría crítica, la teoría de sistema y el post-modernismo.

Se observa entonces que se reconocen debilidades a las teorías del riesgo. Al respecto, Stalker (2003:215), citando a Lupton (1999), afirma que nadie niega la existencia que vivimos en una sociedad de riesgo, aún si existen matices en la manera de percibir y medir dichos riesgos y plantea que las teorías sociales que se refieren a la intervención social y a la acción práctica enfatizan la necesidad que las ciencias sociales adopten una actitud crítica en cuanto a la interpretación del riesgo y cuestionen las actuales respuestas para enfrentarlo.

Prolongando la visión crítica de Douglas (1992) hacia el concepto de riesgo, surgen otros cuestionamientos. Se argumenta que el neo- 
liberacionalismo ha utilizado la teoría del riesgo para atacar el enfoque de la sociedad del bienestar (Culpitt, 1999) y desacreditar el trabajo social (Parton, 1996:98; Stalker, 2003:216).

Al vincular la problemática del riesgo social con su utilización por actores económico-políticos, se introduce entonces una nueva dimensión a la temática. Balardini (2002:22), psicólogo argentino, aplicando la reflexión sobre el riesgo social a la realidad de la niñez y de la juventud, entiende la gestión del riesgo en término de gestión política del riesgo desde las políticas de Estado hacia la niñez y la juventud.

Noceti (2005) aborda también la dimensión política de la noción de riesgo e introduce la noción de Política Social Estatal. Lo define como un conjunto de instrumentos estatales, esto es, acciones-omisiones, bienes y servicios, dirigidos a incidir sobre las condiciones de vida de la población, y sobre el orden social existente (Noceti, 2005:17).

Noceti profundiza su crítica y plantea que identifica en Argentina dos tipos de Politica Social Estatal. Por un lado las políticas de seguridad social y por otro las políticas de asistencia pública. Las primeras están relacionadas a la protección de aquellos grupos que poseen un trabajo estable, incluidos en el «mercado laboral formal». Se supone que estos sectores poseen una capacidad contributiva hacia el Estado -mediante gravámenes-y por tal motivo obtienen una determinada protección social. En ese caso, «el beneficiario es un derecho que el individuo posee dada su contribución al financiamiento de aquel» (Isuani, 1985:73).

Las segundas refieren a grupos contributivos que se encuentran por fuera del mercado laboral. Considerados población vulnerable (desempleados, ancianos, discapacitados, niños, enfermos) que se encuentran fuera del sistema de seguridad social. En este caso, la asistencia «reviste un carácter caritativo, una dádiva a los sectores más necesitados» (Isuani, 195:73). Esta dualidad acarrea desigualdad en palabras de Isuani estamos ante «la configuración de población de primera» vinculado a sectores cubiertos por la seguridad social y «población de segunda» relacionado con sectores que caen bajo el paradigma de la política de asistencia pública. En lugares como América Latina, donde el sector informal es cada vez más creciente y los recursos con que cuenta la asistencia pública son menores que los existentes dentro del esquema de seguridad social, la desigualdad es mayor.

Concluye que esta situación da lugar a un modelo neoliberal de Políticas Sociales Estatales, o sea donde el Estado se convierte en «un 
Estado de Bienestar Híbrido» según modelos residuales al estilo de EE.UU. e Inglaterra donde la focalización de políticas resulta conducente con la idea de pobres merecedores, la ruptura de pactos solidarios entre clases sociales y la creciente brecha socioeconómica entre trabajadores y no trabajadores (Noceti, 2005:18). ${ }^{4}$

La gestión del riesgo en este caso significa «la transformación integral de ciertos principios de organización de la sociedad» (Noceti, 2005:18) y una estrategia determinada. «Esto supone la construcción de toda una serie de consensos intra sistemas de partidos, consensos en el aparato estatal y consensos con otros actores, con actores sociales, fundamentalmente» (Balardini, 2000:22).

Al referirnos a política públicas, Balardini aborda el tema en término amplio, en el sentido gramsciano, incluyendo sociedad política y sociedad civil que construye el consenso más complejo. Balardini (2000:22) plantea que, de esta concepción amplia, se debe traducir en integralidad y coordinación que supera la dimensión meramente discursiva, alcanzar un consenso entre partido para legislar, y luego, alcanzar la transformación de la cultura organizacional de la administración pública y de la relación de los sujetos sociales con el aparato estatal, buscando avanzar en la construcción de acuerdos graduales y desde ellos, ir avanzando hacía algún grado de coordinación y progresivamente hacia una política integral e integrada en el sujeto (Balardini, 2000:22-23).

La gestión del riesgo se traduce luego en implementación de las políticas a diversos beneficiarios: individuales, familiares, grupales, territoriales. Estos focos de acción se articulan en distintos planos o ámbitos de la vida social: el plano societal, o sea aquellas dimensiones de la vida social que poseen carácter específico para cada Estado nacional; el plano comunitario, o sea el ámbito de la convivencia social dentro de sus diversos subsistemas; y el plano institucional, o sea el modo que el Estado y la sociedad civil se organizan para la distribución de oportunidades, medios y recursos, generando espacios estruc-

4 En Chile también se efectúan cuestionamientos al Programa de Administración Directa de Sename (bajo el cual operan los centros de internación cerrados y semi-cerrados para los adolescentes infractores de ley que deben cumplir condenas). «Entre otros aspectos, se evalúa insatisfactoriamente la asignatura de recursos entre regiones y componentes, ya que no intervienen criterios técnicos tales como gastos unitarios de atención, gasto por plaza o por tipo de centro, sino que son asignaturas de acuerdo a presupuesto histórico» (Libertad y Desarrollo, 2007:1). 
turados de participación y canalización de necesidades (Pérez-Luco, $\mathrm{S} / \mathrm{F}: 15-16)$.

De esta manera, la intervención profesional se traduce en distintos planos: el plano psicosocial, o sea acciones centradas en los individuos para modificar las relaciones abusivas, los comportamientos destructivos o la desadaptación social que los aquejan; el plano comunitario donde ser promuevan acciones de desarrollo para dinamizar el crecimiento y el desarrollo de la comunidad; el plano institucional donde se realizan acciones para brindar el apoyo que necesitan distintos individuos o grupos sociales en sus respectivos procesos de desarrollo (Perez-Luco, S/F:18). Se observa también que, en muchos casos, estas múltiples acciones no siempre logran una articulación y coordinación adecuadas entre sí.

\section{ENFOQUES EN TORNO A LA GESTIÓN DEL RIESGO SOCIAL}

Mientras Noceti abordaba la gestión del riesgo social como «la transformación integral de ciertos principios de organización de la sociedad» (Noceti, 2005:18) y Balardini (2000:22) como «la construcción de toda una serie de consensos intra sistemas de partidos, consensos en el aparato estatal y consensos con otros actores, con actores sociales, fundamentalmente» (Balardini, 2000:22); Stalker (2003:218) enfoca la gestión del riesgo como acciones elaboradas por los agentes sociales para minimizar los desenlaces negativos de los riesgos que emergen del presente modelo de desarrollo.

Varios autores (Guerney, 2000a; Langan, 1999; Parsloe, 1999; Stevenson, 1999; citados por Stalker, 2003:217) plantean que en la actualidad hace falta un sólido modelo de gestión social del riesgo, destacando la pluralidad de estrategias de acción en cuanto a la gestión del riesgo.

Al respecto Stalker (2003:218-219) confronta estudios de Alaszewski y Manthrope (1998) con estudios de Hood y otros (2001) para demostrar dicha pluralidad de estrategias y acciones así como el debate en torno al tema.

Alaszewski y Manthorpe (1998), haciendo eco de un estudio de Douglas (1992), advierten que poca atención se ha dado a la manera como las instituciones sociales hacen la gestión del riesgo. Revisando una serie de estudios organizacionales clásicos, en particular con relación a la gestión de agencias de beneficencia, desde una visión de ries- 
go, concluyen que la burocracia tradicional y las agencias que centran su acción sobre la acción del personal en lugar de la acción participativa, tienden a depender de la consejería del experto, castigar los errores y buscar controlar el entorno mediante una acción de prevención y anticipación del riesgo. Por el contrario, las agencias que descentralizan su acción en la participación de los usuarios son más flexibles, delegan decisiones y valores el juicio individual. Propician mayor participación en las decisiones, aprenden de los errores y tratan de desarrollar una respuesta rápida para enfrentar los problemas en lugar de gastar recursos en tratar de anticiparse a los riesgos específicos.

Los estudios de Hood y otros (2001:1992) cuestionan las conclusiones de Alaszewski y Manthorpe (1998), argumentando que no hay tal cosa que la sociedad del riesgo sino sólo una manera diferentes regímenes de regular el riesgo (2001:171). Observan una pluralidad de orientaciones en el seno de las instituciones sociales y las resume en las siguientes alternativas: o la anticipación del riesgo o la rapidez para enfrentarlo; o el castigo a los profesionales por sus errores o la tolerancia basada en una cultura del aprendizaje a partir de los errores; o el uso de ingeniería social ortodoxa para manejar el riesgo o la búsqueda de alternativas nuevas; o un enfoque de reducción de los riesgos a corto plazo o una estrategia a largo plazo; o la toma de decisión por parte de las autoridades mayores guiados por expertos o la participación de los usuarios en la toma de decisión para la gestión de los riesgos; o una aproximación de la regulación basada en procesos cualitativos o en resultados cuantitativos.

Gurney (2000a) plantea entonces que se puede graficar las tensiones entre los diversos orientaciones para enfrentar el riesgo social en un continuo entre un polo más defensivo y de corto plazo, centrado en el control del riesgo por la autoridad pública, y un polo más audaz, que se arriesga a brindar participación y empoderamiento a las víctimas y victimarios, $\mathrm{y}$ que favorece una aproximación profesional de largo plazo centrada en remediar a las necesidades sociales y reparar los daños. Stalker (2003:219-223) utiliza dos términos para confrontar las dos orientaciones «risk-avoidance» y «risk-taking», lo que se describe a continuación.

\section{a) El polo «Risk-Avoidance»}

Se puede ilustrar este enfoque para enfrentar el «problema de la juventud» (Muncie, 2004) mediante políticas y prácticas marcadamente 
influenciadas por el paradigma de la prevención y control público del riesgo por parte del sistema de Justicia (Goldson y Muncie, 2006a, 2006b; citados por Case, 2007:91) definido en término específico de prevención y control de la criminalidad y delincuencia mediante estudios de caracterización juvenil y de su entorno. Consiste en introducir un ethos de gestión y de control que reemplaza la preocupación anterior del bienestar y de la justicia social (Muncie y otros, 2002), así como en priorizar la auditoria estadística del riesgo de delincuencia en lugar de un monitoreo de los procesos de la rehabilitación (Annison, 2005; Smith, 2006; citados por Case, 2007:92).

Según Case (2007:92), este enfoque de auditoria se plasma en un modelo pragmático de prevención del crimen que usa el survey para identificar, desde los aspectos claves de la vida de una persona joven (familia, escuela, comunidad, rasgos psicoemotivos del individuo), los factores que incrementarían la eventualidad de la ocurrencia de una falta o delito (factor de riesgo), o que disminuirían su eventualidad (factor de protección). Esta prevención-control del crimen y de la delincuencia, basada sobre los factores de riesgo, sustituye el enfoque de abordar la delincuencia mediante la mejoría de las oportunidades de desarrollo para todos y la búsqueda de la reparación y la rehabilitación de los infractores de ley, lo que reorienta la justicia dirigida a la juventud hacia la búsqueda de evidencias de factores de riesgo y la elección de una estrategia de protección mediante el control público.

Stalker (2003:219) cita a Douglas (1986, 1992), Beck (1992), Kemshall y otros (1997) argumentando que la preocupación por prevenir el riesgo social mediante el énfasis en el control está relacionado con la pérdida de la fe en la ciencia, el trabajo social, el conocimiento y el poder jerárquico tradicional. Beck considera que la base normativa de la sociedad del riesgo es la seguridad y que la utopía es negativa y defensiva. Kemshall y otros (1997:223) argumentan que la política pública actual está muy preocupada de investigar lo que anduvo mal en una situación para acusar a los responsables, lo que provoca una actitud defensiva por parte de los interventores sociales, aún si no es la decisión más apropiada (Parton, 1998).

En este polo se ubicaría quienes favorecen la privación de libertad para jóvenes que infringieron la ley en lugar de programas semicerrados o internación provisoria. Cabe mencionar que surgen múltiples críticas al impacto de la privación de libertad: mutilación del Yo, exacerbación del control sobre el tratamiento, desproporción adaptativa, 
desvaloración de la actitud creativa y rutinización de la experiencia, adaptación a las dinámicas de violencia y las formas de vida en el encierro, «desnormalización» del desarrollo afectivo, sexual y físico, desvinculación de las redes y contextos de integración social, reincidencia (Valdebenito, 2007).

De hecho, la crítica al enfoque del paradigma de la prevencióncontrol de los factores de riesgo cobra gran importancia en la literatura sociológica contemporánea. Al respecto, Stalker (2003:220) se refiere a Tanner (1998:15-28) que sugiere que este enfoque se substituye a la preocupación de las necesidades sociales de la gente, a la vez en las políticas de salud, de beneficencia y de previsión social, y que la atención social se queda a la superficie de los problemas, más centrados en la seguridad, las normas y los procesos de retención. Califica dicha práctica de culto a los procedimientos donde se observa poca referencia a los valores y a los procesos de restauración. Kemshall (2002:30) plantea que las políticas sociales obedecen a una fuerte priorización donde los controles de selección y de servicio a corto plazo prevalecen sobre el trabajo estratégico y a largo plazo. Se advierte que esta política a la larga puede ser más costosa. ${ }^{5}$

Se observa también un cuestionamiento al enfoque mismo, acusándole de falta de validez. Se considera inadecuadas las intervenciones basadas en la mera «evidencia» establecida por la investigación cuantitativa elaborada desde el paradigma de la prevención-control del riesgo. Las críticas afirman que este enfoque es teórica y metodológicamente débil, estático, limitado en su perspectiva (Case, 2007:91) y a menudo manipulado para sacar ventajas mediante políticas de corto plazo (Hughes y otros, 2002; Bateman y Pitts, 2005).

Stalker (2003:221) destaca como Waterson (1999) advierte acerca de cómo esta actitud de control del riesgo en trabajo comunitario, instituciones sociales y actividades de beneficencia se traduce por una gran preocupación por medidas de seguridad en el trabajo, una racionalización de los recursos precarios y una reducción del trabajo preventivo. Dada esta preocupación, se reduce el trabajo a largo plazo. En cuanto a los servicios dirigidos a la niñez en dificultad. En esta crítica

5 La criminóloga chilena Doris Cooper afirma que en tiempos electorales, los políticos compiten en nuevas y más duras medidas represivas. Plantea que la pobreza y el desempleo generan siempre más delincuentes de los que es posible encarcelar. Y que no sirve para nada construir más cárceles (www.lanacion.cl, 2 de octubre de 2005). 
al riesgo como control, Stalker (2003:222) cita también a Parton (1998) que argumenta que la atención a la niñez en dificultad opera en una zona mal definida entre la familia (mundo privado) y el mundo público, territorios ambiguos y cuestionados. Una legislación reciente en el Reino Unido se basa en principios duales, pero conflictivos, de cooperación y apoyo a las familias así como de protección y prevención de riesgo. Parton (1998) denuncia que en contextos de pobreza y familias socialmente excluidas, los recursos sociales han disminuidos, así como las actividades de prevención. Los esfuerzos se centran en identificar los casos de gran riesgo, utilizando criterios legales y evidencia forense. $\mathrm{La}$ auditoría ha reemplazado la confianza en el trabajo social.

En esta misma línea, Stalker (2003:217) aporte al debate refiriéndose a Parloe (1999) que argumenta que la pobreza, la vivienda insalubre y la enfermedad pueden ser tan dañinas como el abuso y a Langan (1999) que señala como los estudios han dado poca atención a las enfermedades mentales y a los riesgos que corren individuos en situaciones socioeconómicas de precariedad, en particular la infancia, la juventud y la mujer. Se advierte que múltiples riesgos surgen del entorno (Wilson, 1994). Parloe (1999) llama la atención en la importancia de elaborar un modelo de gestión social del riesgo basado sobre factores del ambiente y redes sociales.

El concepto de Seguridad Humana rescata en parte esta propuesta y aborda la cuestión del riesgo desde un enfoque más amplio (PNUD, 1998:80), aún si el concepto de Seguridad Humana no se restringe a caracterizar el riesgo infantil y juvenil. Este concepto se define mediante siete categorías de amenazas a superar: peligros contra la seguridad personal (seguridad respecto a la violencia física y psicológica); amenazas a la seguridad económica (ingreso básico), a la seguridad alimenticia (acceso al alimento), a la seguridad en salud (acceso a un pleno desarrollo físico), a la seguridad ambiental (medio físico saludable), a la seguridad de la comunidad (participación en un grupo, una familia que brinda identidad cultural un conjunto de valores), a la seguridad política (sociedad donde se respetan sus derechos).

Sin embargo Case (2007:92) no niega la importancia de esta aproximación de prevención/control al riesgo pero hace recomendaciones al respecto: se debería ampliar la práctica cuantitativa tradicional de prevención/control de factores de riesgo e introducir una rutina de monitoreo de los procesos de cuidado de la infancia y juventud en situación de dificultad, incluyendo en la muestra una población relati- 
vamente poco tomada en cuenta: la misma juventud y los profesionales y personas de trato directo en la protección y la responsabilidad juvenil.

\section{b) El polo «Risk-Taking»}

Si el primer polo de intervención enfatiza el control del riesgo, en el polo opuesto se considera al beneficiario de los servicios sociales como un ciudadano activo con derechos y responsabilidades, valorando sus habilidades. Se deja de considerar a los expertos como los únicos actores válidos (Gurney, 2000b). Se enfrenta la prevención del riesgo tomando riesgos medidos, educando al usuario a tomar responsabilidades y contribuir activamente a su empoderamiento y desarrollo. En la literatura se considera que el aceptar de correr riesgo en las actividades de gestión del riesgo es considerado razonable, legítimo y apropiado (Brearley, 1982a; 1982b; Kemshall y otros, 1997; Waterson, 1999; citados por Stalker, 2003:223).

En este polo se ubican los que privilegian las penas no privativas de libertad para quienes han infringido la ley. Al respecto se plantean las siguientes ventajas de esta estrategia: limita los costos económicos, personales y sociales de la privación de libertad, favorece la atención personalizada del joven por medio de la supervisión del delegado y de un plan de intervención individualizado, ofrece una gama de intervenciones tendientes a fortalecer las habilidades del joven y su familia con miras a auspiciar la no reincidencia (Valdebenito, 2007).

Según este enfoque, los individuos ganan más control sobre sus vidas y participan en la elaboración de su agenda de acción en lugar de responder a las meras prescripciones de los profesionales, enfoque que es el eco de una larga tradición en intervención social en la línea de la educación del individuo a la autonomía (Stalker, 2003:223).

Balardini (2000:23) se ubica en este polo al centrar su enfoque en la participación de los sujetos (los jóvenes) en las políticas de juventud, lo que determina diversos estilos de gestión y la construcción de diversos sujetos, según si este modelo sea «para», «por», «con», o «desde» la juventud.

Recientemente, existe un interés creciente en la intervención social por el concepto de resilencia afirma Stalker (2003:226). Al referirse al tema, cita a Jackson (2000:296) que define resiliencia como «una interacción entre riesgo y factores protectores..., lo que puede interrumpir y revertir lo que de otra manera conllevarían procesos de da- 
ño». Cita también a Fraser y otros (1999) que se refieren a la resiliencia como características individuales, factores familiares o circunstancias extra-familiares que contribuyen a enfrentar mejor una situación de riesgo.

En este polo se ubican también las experiencias centradas sobre el trabajo individual y comunitario que insisten en la importancia de abordar la prevención desde un trabajo cultural con las familias y la niñez en situación de dificultad para fortalecer sus capacidades de asumir con madurez sus diversos roles (familiares, económicos, culturales) en este cambio global que se vive. Se conjuga con programas socioeconómicos para incrementar las oportunidades de trabajo para las familias y jóvenes vulnerables y asegurar su inserción laboral, cuidando en todo momento la rehabilitación de las jóvenes que sufrieron daños causados por atropello a los derechos o por conducta desviada.

En esta perspectiva se enfatiza la importancia de movilizar y coordinar a toda la comunidad y sus diversas instituciones (la familia, la economía, la escuela y el colegio, las iglesias, el municipio, las instituciones sociales) para asegurar un auténtico desarrollo de la infancia y de la adolescencia. Este enfoque propicia la promoción de una acción intersectorial en el cual los distintos poderes del Estado y de la comunidad tengan el papel central en la generación de condiciones para que los niños y niñas y adolescentes sean sujetos de derecho y alcancen su pleno desarrollo (Bourque y otros, 2007:6).

En coherencia con esta problemática, se plantea que la protección y promoción de los derechos de la infancia y juventud en riesgo no se solucionan solamente por políticas sociales, sino debe incluir un acompañamiento psicoeducativo que se preocupe de la niñez y juventud más desamparadas para impedir que entren inexorablemente en el círculo vicioso de la marginalidad, que convierten la falta de trabajo y de educación, o la desorganización de la familia, en incapacidad de llevar a cabo un esfuerzo personal o en encierro en una banda más o menos delincuente (Touraine, 1990:5).

Las estrategias se centran en establecer una relación de confianza, ayudar a reparar el daño causado por el abuso y el rechazo, fortalecer sus capacidades para superar su frustración, su encierro narcisista y sus adicciones destructivas. Este apoyo busca que esta niñez y juventud en dificultad alcancen ser actores sociales responsables de su propia vida, capaces de construir proyectos de vida, tener relaciones sociales, elegir las redes sociales las más apropiadas para su bienestar, capaces de 
descubrir las oportunidades del medio y de transformar su entorno social para realizar sus proyectos personales (Touraine, 1990:6).

Cabe recordar que el enfoque de la psicoeducación ha transformado las prácticas sociales hacia la infancia y juventud en situación de dificultad. Desroches y Dionne (2008:149-150) recuerdan el contexto del desarrollo de la psicoeducación en el contexto social de Québec, Canadá. Se afirma que, anterior a los años sesenta, los adolescentes que habían cometido delitos eran ubicados en prisiones de adultos o en reformatorios muy represivos o severos. Los niños huérfanos, los niños que tenían retrasos intelectuales, los niños y los adolescentes que tenían dificultades de salud mental, eran todos ubicados en grandes hospitales psiquiátricos. Se observa en Québec como, con la reforma de los años sesenta llamada la «Revolución Tranquila» dirigida a la educación y a los servicios sociales, se ha reducido el tamaño de las instituciones sociales de cuidado a la infancia y especializado la atención a los niños, incorporado un enfoque de protección y de rehabilitación, elaborando un modelo terapéutico e instrumentos pedagógicos, organizando un ambiente físico más apropiado, articulando un apoyo científico para la acción educativa y una formación profesional de los educadores (Renou, 1989; citado por Desroches y Dionne, 2008: 149-150).

Esta problemática plantea entonces que una política integral para la infancia y la juventud debe priorizar a quienes están con menos oportunidades (Touraine, 1990:5). Debe mejorar las políticas de bienestar y de justicia social para las familias más pobres y asegurar oportunidades de trabajo para la juventud de sectores populares marginales (Muncie y otros, 2002). Debe también valorar el acompañamiento psicoeducativo para asegurar el «desarrollo personal integrado» que «incrementa en los jóvenes la capacidad de comportarse como actores sociales» (Touraine, 1990:4) en la sociedad actual. Las orientaciones buscan fortalecer la Seguridad Humana, o sea los mecanismos sociales que hagan posible la acumulación de capacidades que las personas puedan lograr en el tiempo gracias al sano equilibrio o complementariedad entre las distintas condiciones sociales (PNUD, 1998:18).

En esta búsqueda de un enfoque integral en la gestión del riesgo se acude también a una referencia a los valores. Dado que nuestro tiempo conlleva una crisis de sentido, o sea una puesta en tela de juicio de todas las certezas y el cuestionamiento de las identidades, cambios cada vez más intensivos en las estructuras familiares y una creciente tendencia hacia el deseo de seguridad, surgen conflictos en torno a las 
distintas soluciones propuestas: los fines y los contenidos de la vida compiten entre sí, de manera que, en este contexto las orientaciones que se pretenden eficaces tendrán que responder al desafío de hacer compatibles ciertos conceptos de la vida que resulten válidos para el individuo con otras indicaciones que apoyen la condición comunitaria de la sociedad.

De allí que la búsqueda de soluciones exige también procesar el sentido de lo que sucede en su vida y entorno. En este sentido se reconoce la importancia de las instituciones intermedias (familia, escuela, iglesias, ONGS) para ayudar al individuo a procesar sus conflictos subjetivos de sentido (Berger y Luckmann, 1997:1, 124) y, más que todo, apoyar a quienes fueron más atropellados por la vida a fin de que alcancen a resignificar su vida y alcanzar un nuevo interés a vivir.

En esta perspectiva de la búsqueda de sentido y empoderamiento, cabe destacar experiencias emergentes promisorias que incorporan la dimensión valórica, o sea prácticas sociales de comunidades de fe que acompañan a familias y niños en situaciones de riesgo. Desde la fe en el mensaje de Jesús, y con el apoyo de profesionales, estas comunidades buscan con las familias y los niños en dificultad como enfrentar con esperanza los riesgos de su situación y las dimensiones trágicas de su vida tales como la violencia, el sufrimiento, la muerte, la culpabilidad, el rechazo y la soledad, la cesantía, la marginalidad. Su estrategia, si bien contempla dimensiones espirituales (énfasis en los valores, oración, lectura bíblica y resignificación de su experiencia,), incluye también una vida comunitaria activa que emprende una acción solidaria y un acompañamiento integral a la niñez en dificultad, buscando la reparación del daño, la creación de oportunidades laborales, el fomento de la reintegración familiar y la vinculación a redes sociales.

\section{CONCLUSIONeS}

Este artículo observa como las grandes transformaciones (económicas, políticas y culturales) impactan en la vida cotidiana de la gente, dificultando a la familia para gestionar sin desintegrarse los desafíos que se le presentan, y afectando también a la niñez y juventud. Éstas no solamente incorporan pautas y valores como drogadicción y alcoholismo, sino también llegan a la ruptura con el proyecto familiar y la construcción de lazos intergeneracionales. Ello plantea un gran desafío a la sociedad y al Estado para implementar instrumentos públicos y 
comunitarios para asegurar la inclusión social de toda su niñez y juventud y la reinserción social de aquellos que han sido vulnerados en sus derechos.

La exploración de la literatura sobre la gestión del riesgo y la incertidumbre en la intervención social con niñez y juventud en situación de dificultad revela que, si bien el concepto de riesgo fue vinculado a la probabilidad de ocurrencia de eventos y sus consecuencias en término de ganancia y pérdidas, se observa que dicho concepto ha evolucionado y hoy en día está utilizado habitualmente para referirse a un desenlace negativo o incierto.

En cuanto a los esquemas teóricos, se observa que en sociología y antropología los estudios enfatizan dimensiones culturales y políticas del riesgo, sin negar la existencia de riesgos reales.

Esta conciencia creciente del riesgo que produce nuestro sistema socioeconómico conlleva una tendencia a perder fe en los expertos y en la intervención social, y una tendencia de los Estados en incrementar medidas de control social, lo que se revela en un debate académico y profesional en torno a las estrategias de gestión del riesgo. Se observa el avance político-administrativo de la tendencia del «risk-avoidance», o sea aquella que busca anticipar y controlar la ocurrencia del riesgo, sobre la «risk-taking», o sea aquella que apunta a construir capacidades en los sujetos sociales mediante un trabajo cultural con las familias y la niñez mediante la entrega de responsabilidades, aún si esta estrategia conlleva un porcentaje de riesgo (Case, 2007:100; Stalker, 2003:211).

A pesar de una tendencia hacia la seguridad enfocada en el control social, se observa también una tensión hacia la búsqueda de estrategias más integrales de gestión del riesgo centradas en un apoyo a la niñez y juventud vulneradas en sus esfuerzos de reconstrucción de un sentido de su vida, de un entorno afectivo positivo significativo, y de oportunidades materiales y laborales que le permitan insertarse en la sociedad. Esta búsqueda de estrategias integrales no excluyen en sí una referencia a la trascendencia. En este proceso, las instituciones intermedias (la familia, la escuela, las iglesias, las ONGS, etc.) pueden brindar su contribución como comunidades de sentido, de vida y de fe, y así evitar que la identidad de la persona individual y la coherencia intersubjetiva de la sociedad se vean amenazadas o inclusive destruidas por una modernidad acosada por la crisis (Berger y Luckmann, 1997:125).

Sea cual sea la estrategia de gestión adoptada, Valdebenito (2007) recalca lo desafiante de la reinserción social de la niñez y juventud 
vulneradas en sus derechos. Con relación a la reinserción escolar de la niñez, este desafío se relaciona con el rezago del joven en su desempeño escolar y la negatividad de su actitud frente a la escuela, así como con las actitudes negativas de muchos educadores en incorporar estos estudiantes «irregulares» en su sistema educativo para alumnos «regulares». La reinserción social tiene que ver también con la dificultad de la reeducación de las condiciones psicológicas de la niñez y juventud vulnerada: la impulsividad, las dificultades para evaluar riesgos, la disminuida capacidad de autocontrol y la resolución de tensiones mediante hábitos de violencia. La reinserción social tiene también que ver con las características del entorno: el grupo de pares, los estilos de supervisión parental, las condiciones socioeconómicas de vida, las oportunidades de trabajo, los lazos sociales y laborales, el grado de criminalización de la condición étnica y de la marginalidad social, las competencias de las instituciones públicas y privadas que brindan apoyo a la niñez y juventud en dificultad.

Dado lo desafiante de la reinserción social de la niñez y juventud vulneradas en sus derechos, Cohen y Franco (2005:10) nos advierten de la importancia de la gestión de las políticas sociales. Afirman que, tanto en el pasado como en la actualidad, los programas sociales ejecutados en América Latina han estado lejos de alcanzar sus objetivos. A pesar de un incremento en el esfuerzo del Estado latinoamericano, ${ }^{6}$ se observa poca eficacia en el desarrollo de los productos que generan, lo que se traduce a la vez, en un magro efecto en la situación de bienestar de las personas a las que se dirigen.

Atribuyen el hecho a las notorias deficiencias en la identificación de los grupos a los que estos programas estaban orientados, al mal manejo de los recursos disponibles y a una ineficaz generación de los productos con los que se pretende cubrir las carencias de la población destinataria. Se plantea así la exigencia de pensar no sólo en el diseño de las políticas sino también en su gestión, o sea en la formulación, evaluación, implementación y monitoreo de las políticas, programas y proyectos sociales, y de las condiciones institucionales y organizacionales necesarias para obtener resultados adecuados. Todo ello se engloba bajo la expresión gestión social.

6 En Chile se recalca que siguen precarios los recursos destinados a la prevención, protección y reinserción social de la niñez y juventud (Libertad y Desarrollo, 2007). 
En coherencia con el planteamiento de Cohen y Franco, cabe rescatar también el aporte de la psicoeducación que insiste en cuidar la estructura y el proceso educativo de reparación, rehabilitación y reinserción social. Más aún, enfatizando el punto de vista del enfoque valórico, se puede decir que la reparación y la rehabilitación no solamente exigen aportes cognitivos y de control. Se considera que el daño causado por la vulneración de los niños exige de los educadores, además de una formación profesional, una madurez emotiva y una capacidad de empatía para establecer un «rapport» afectivo de calidad con los niños y niñas vulnerados. Son condiciones necesarias para que los educadores puedan colaborar con ellos a resignificar su vida, construir un horizonte de esperanza que les motive a emprender un nuevo curso de acción y adquirir las capacidades necesarias para superar los daños de relaciones abusivas, los impulsos autodestructivos y la mesadaptación social.

Las advertencias de Cohen y Franco, así como del enfoque de la psicoeducación y del enfoque de los valores, recalcan lo esencial del conocimiento, sistematización y evaluación de los trabajos empíricos que se realizan en este campo. Si bien existen avances en los estudios teóricos en esta temática (Jaeger y otros, 2001), cabe destacar que pocos trabajos empíricos han sido realizados sobre gestión del riesgo social (Macdonald y Macdonald, 1999; citado por Stalker, 2003:228), lo que exige promover la sistematización de experiencias en este campo y su análisis con rigor teórico-metodológico para relacionar mejor los procedimientos de los profesionales ya señalados (el modelo terapéutico, la organización y el ambiente físico, los instrumentos pedagógicos utilizados, la formación específica de los educadores, los recursos financieros) y los resultados alcanzados, sin dejar de incluir el estudio de los factores condicionantes que surgen del contexto. Ello conllevará a enfrentar mejor los desafíos actuales de la niñez y de la juventud y promover políticas sociales e instrumentos de intervención social más pertinentes.

TEMUCO (CHILE), SEPTIEMBRE 2007

RECIBIDO: NOVIEMBRE 2007

ACEPTADO: MARZO 2008

Patrick Donovan, Ximena Oñate, Gonzalo Bravo y María Teresa Rivera agradecen a la Dirección de Investigación de la Universidad de La Frontera por su patrocinio a nuestra investigación (Proyecto $\mathrm{N}^{\circ} 120507$ ) titulada «Iniciativas sociales y desarrollo local: monografías de intervenciones sociales vinculadas a niños y niñas menores de 18 años en dificultad» (Temuco, Chile; 2005-2006). 


\section{REFERENCIAS BIBLIOGRÁFICAS}

AlaszewSKi y Manthrope (1998): «Welfare Agencies and Risk: the Missing Link», Health and Social Care in the Community 6 (1):4-15.

ALBUQUERQUE, FRANCISCO (1999): «Manual del agente de desarrollo local». Santiago: Ediciones SUR.

AnNison, J. (2005): «Risk and Protectión». T. BAteman and J. PITtS: The RHP Companion to Youth Justice. Lyme Regis: Russell House.

Arocena, J. (2001): El desarrollo local, un desafio contemporáneo. Montevideo: Universidad Católica del Uruguay.

BALARDINI, S. (2000): «De los jóvenes, la juventud y las políticas de juventud». Última Década $\mathrm{N}^{\circ} 13$. Valparaíso: Ediciones CIDPA.

Bateman T. and J. PitTs (2005): The RHP Companion to Youth Justice. Lyme Regis: Russell House.

BECK, U. (1992): Risk Society: Towards a New Modernity. London: Sage.

BERGER, P. y T. LUCKMANN, (1997): Modernidad, pluralismo y crisis de sentido, la orientación del mundo moderno. Barcelona: Paidós.

Bourque, D., Y. Comeau, L. Favreau et L. FréChette (2007): L'organisation communautaire, fondements, approches et champs de pratique. Québec: Presses de l'Université du Québec.

BREARLEY, P. C. (1982): Risk in Social Work. London: Routledge and Kegan Paul.

_ et al. (1982): Risk and Ageing. London: Routhledge an Kegan Paul.

CASE, S. (2007): «Questionning The 'Evidence' of Risk that Underpins Evidence-Led Youth Justice Interventions». Youth Justice 7. London. Sage.

CASTELlS, M. (1999): «Globalización, identidad y Estado en América Latina». Temas de Desarrollo Humano Sustentable. Santiago: PNUD.

COHEN, E. y R. FrANCo (2005): Gestión social, cómo lograr eficiencia e impacto en las politicas sociales. Santiago: Siglo XXI.

COOPER, D. (2005): «Los políticos están locos», Santiago, La Nación, Domingo 2 de octubre.

Colectivo (2004): Un Chile apropiado para los niños. Santiago: Sename.

Colectivo (2005a): Manual de procedimiento para la protección de los derechos de la infancia y adolescencia. Santiago: Sename.

Colectivo (2005b): «Los efectos de los programas del Sename en la integración social de los niños y niñas». Estudio 6. Santiago: Sename.

Colectivo (2006): Catastro de oferta programática de la Red Sename. Santiago: Sename.

CulpitT, J. (1999): Social Policy and Risk. London: Sage.

DeSROCHERS, C. y J. DionNe (2008): «Psicoeducación». Temuco: Universidad de La Frontera y Universidad de Québec en Outaouais. 
Donovan, P., G. Bravo, X. OÑate y M. T. Rivera (2006): «Intervención con niños y niñas menores de 18 años en situación de dificultad psicosocial en Temuco: la visión de los actores ». Ponencia en " Congreso internacional de psicoeducación niñez y juvetud en dificultades psicosociales: escenario y desafíos en las prácticas psicoeducativas». Temuco. Universidad de La Frontera.

Douglas, M. (1992): Risk and Blame: Essays in Cultural Theory. New York: Routledge.

FARRINGTON, D. P. (1998): «Predictors, causes, and correlates of male youth violence». M. TOURY and N. MORRIS (eds.): Crime and Justice: an annual review of research Vol. 24. Chicago: University of Chicago.

FoucAult, M. (1995): La microfisica del poder. Buenos Aires: La Piqueta.

Fraser, M. W., J. M. RichMAN and M. J. GALINSKY (1999): «Risk, Protection and Resilence: Towards a Conceptual Framework for Social Work Practice». Social Work Research 23 (3).

GAETE, A. y R. LARA (2007): «Panorama nacional y regional de la delincuencia infanto-juvenil». Seminario-Taller «Seguridad pública y delincuencia infanto-juvenil a nivel nacional y regional». Temuco: Universidad de La Frontera.

GIDDENS, A. 2000: Sociología. Madrid: Alianza.

GOLDON, B. and J. MUNCIE (2006a): «Rethinking Youth Justice: Comparative Analysis, International Rights and Research Evidence». Youth Justice 6 (2). and - (eds.) (2006b): Youth Crime and Justice: Critical Issues. London. Sage.

GotTFredson, M. R. and T. HiRSCHI (1990): Causes of Delinquency. Berkeley: University of California Press.

GurneY, A. (2000a): «Risk Management». DAvies (ed.) (2000): The Blackwell Encyclopedia of Social Work. Oxford: Blackwell.

(2000b): «Risk taking». M. DAVIES (ed.) (2000): The Blackwell Enciclopedia of Social Work. Oxford. Blackwell.

HoOD, C., H. RothSTEIN and R. BALDwIN (2001): The Government of Risk: understanding Risk Regulation Regimes. Oxford: Oxford University Press.

Hughes, G., E. MCLAughlin and M. Muncie (2002): Crime Prevention and Community Safety: New Directions. London: Sage.

JACKSON, S. (2000): «Resilence». M. DAVIES (ed.) (2000): The Blackwell Enciclopedia of Social Work. Oxford. Blackwell.

JAEGER, C., O. RENN, E. RosA and T. WeBLER (2001): Risk, Uncertainty and Rational Action. London: Earthscan Publications.

Kemshall, H., N. PARTON, M. WALSh and J. WATERSON (1997): «Concepts on Risk in Relation to Organizacional Structure and Funcioning within the Personal Social Services an Probation». Social Policy and Aministration 31 (3). Sage Publications: London. 
Klein, K., R. Forehand, L. Armistead and P. LONG (1997): «Delinquency during the transitions to early adulthood: Family and parenting predictors from early adolescence». Adolescence 32.

Langan, J. (1999): «Assessing Risk in Mental Health». Social Work №36. London: Jessica Kingsley.

LiBERTAD Y DESARROLlo (2007): «Incendio en centro del Sename: crisis de la nueva institucionalidad penal juvenil». Temas Públicos $\mathrm{N}^{\circ} 844$. Santiago: Libertad y Desarrollo.

LOEBER, R. and D. P. FARRINGTON (1998): «Never too early, never too late: Risk factors and successful interventions for serious violent juvenile offenders». Studies on Crime and Crime Prevention 7 (1).

Luhmann, N. (1993): Risk: A Sociological Theory. New York: Aldine de Gruyter.

LUPTON, D. (1999): Risk. London: Routledge.

MACDONALD, K. I. and G. M. MACDONALD (1999): «Perception of Risk». Social Work $\mathrm{N}^{\mathrm{o}} 36$. London: Jessica Kingsley.

Muncie, M., G. Hughes and E. MCLAughlin (2002): Youth Justice: Critical Readings. London: Sage.

NoceTI, M. B. (2005): «Organizaciones fuertes, presencia y decisión en el devenir de las políticas públicas dirigidas a niños en riesgo social en la Provincia de Buenos Aires». e-latina Vol. 3, №11. Buenos Aires: Universidad de Buenos Aires.

Parsloe, P. (1999): «Introduction». Social Work N³6. London: Jessica Kingsley.

PARTON, N. (1999): «Risk, Advanced Liberalism and Child Welfare: The Need to Rediscover Uncertainty and Ambiguity». British Journal of Social Work 28.

— (1996): «Social Work, Risk and 'The Blaming System'». Social Work No36. London: Jessica Kingsley.

PereZ-LuCo, R. (S/F): «Enfoque psicosocial ecológico, psicología comunitaria en la frontera». Santiago: Sename.

RENOU, M. (1989): «La pasychoeducation: une perspective historique». Revue Canadienne de Psicoeducation 18 (2).

RYAN, T. (1996): «Risk Management and People with Mental Health Problems». H. KeMShall and J. PRITCHARD (eds.): Good Practice in Risk Assessment and Risk Management, Vol. 1. London: Jessica Kigsley.

SAlAZAR, G. y J. PINTO (2002): Historia contemporánea de Chile. V, niñez y juventud. Santiago: LOM.

SAmson, R. J. and J. H. LAMB (1993): Crime in the making: Pathways and turning points through life. Cambridge: Harvard University Press.

SARgent, K. (1999): «Assessing Risks for Children». Social Work No36. London: Jessica Kingsley. 
SMith, R. (2006): «Youth Crime and Justice: Research, Evaluation and Evidence». B. GOLDSON and J. MunCIE (eds.): Youth Crime and Justice. London. Sage.

SprotT, J., J. Jenkin and DooB (2005): «The Importance of Schooll. Protecting At-Risk Youth From Early Offending». Youth Violence and Juveline Justice, Vol. 3, №1. London: Sage.

STALKER, K. (2003): «Managing risk and uncertainty in Social Work, a literature review». Journal of Social Work 3 (2). London: Sage.

Stevenson, O. (1999): «Keeping a Precarious Balance». Community Care (10-16 september), 9.

(1999): «Old People at Risk». Social Work N³6. London: Jessica Kingsley.

TANNER, D. (1998): «The Jeopardy of Risk». Practice 10 (1).

TOURAINE, A. (2001): «El fin de la ola liberal». Desigualdad y globalización, cinco conferencias. Buenos Aires: Facultad de Ciencias Sociales de la Universidad de Buenos Aires.

TOURAINE, A. (1990): «Juventud y sociedad en Chile». Santiago: Unicef. UNICEF (2002): Índice de Infancia, una mirada comunal y regional, Santiago. VALDEBENITO, S. (2007): «Reinserción social». Seminario «Seguridad pública y delincuencia infanto-juvenil a nivel nacional y regional». Temuco: Sename y Universidad de La Frontera.

WARNER, F. (1992): «Introduction». Risk: Analysis, Perception and Management Report of a Royal Society Study Group. London: Royal Society.

WATERSON, J. (1999): «Redefining Community Care Social Work: Needs of Risks Led?». Health and Social Care in the Community 7 (4). London: Sage. 